\title{
Venezia, Biblioteca Nazionale Marciana, gr. Z. 310 (coll. 301)
}

\author{
I. Costantinopoli, metà del XIV sec.; II. Costantinopoli, prima metà del XV sec.
}

Comp., ff. A-B, I-II, 1-288, A'-B'; sul f. A è incollato il foglio, tratto dal catalogo di Zanetti (1741), con il contenuto del manoscritto; sul f. IIv si trova l'ex libris di Bessarione; num. recente nel marg. sup. est: il numero 174 è ripetuto due volte; manca il numero 187. Sono bianchi i ff. 152, 188-192, 204v-205, 262-264. Legatura in marocchino rosso, realizzata durante la campagna del 1722.

Il codice è costituito da due unità: la più antica reca la Syntaxis di Tolomeo e commentarii annessi; risale al secolo XIV ed è stata vergata a Costantinopoli da Isacco Argiro; la seconda, datata al XV secolo, è opera di Bessarione, che ha ampliato con altri testi astronomici la prima sezione.

\section{I. ff. 1-264 (Costantinopoli, XIV sec.)}

Cart.; fil. ff. 4, 6, 7, 9, 10, 11, 13, 18, 21, 22, 24, 31, 32, simile a oiseaux (M/Tr nr. 6617, 1352), ff. 27, 29, 30, 35, 38, 51, 54, 67, 70, 97, 98, 99, 102, 105, 107, 109, 110, 112, 114, 115, 117, 118, 119, 120, 124, 127, 128, 129, 130, 133, 138, 139, 140, 142, 144, 145, 146, 148, 150, 172, 174a, 178, 180, 182, 184, 185, 186, 189, 197, 198 , 199, 200 simile a fruit (M/Tr nr. 4318, 1355-1370), ff. 33, 36, 39, 41, 44, 47, 49, 50, 53, 57, 58, 61, 66, 69, 72, 73, 74, 76, 82, 85, 88, 89, 92, 95, 101 simile a cloche (M/Tr nr. 2846, 1353), ff. 153, 157, 162, 163, 164, 168, 169, 170, 202, 203, 204, 210, 211, 216, 218, 222, 224, 226, 227, 229, 240, 241, 242, 243, 250, 254, 255 , 257, 262, 263, 264 simile a bouc (M/Tr nr. 1641, 1357), ff. 225, 235, 236, 239, simile a cercle (M/Tr nr.

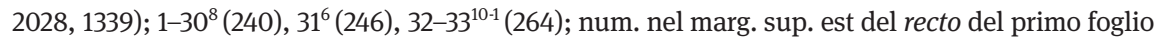
e nel marg. inf. del verso dell'ultimo foglio di ogni fascicolo, numerati da $\alpha$ ' a $\kappa \varsigma^{\prime}$ (f. 202r); mm $293 \times$ 224 = 32 // 213 // $48 \times 19$ // 150 // 9 // 25 // 21; a piena pagina, ll. 41-51, unità di rigatura mm 4; rigatura visibile sul recto di alcuni fogli tracciata a secco, tipo S/L 20C1n (Muz 1-21/0/0/C) (f. 127); mm $294 \times 220$ = 25 // 239 // $29 \times 14$ // 176 // 30; a piena pagina, 11. 49-51, unità di rigatura mm 5; rigatura visibile sul recto di alcuni fogli tracciata a secco, tipo S/L 00A1 (Muz 1-1/0/0/A) (f. 256).

\section{Scrittura}

La sezione è vergata da un solo copista: <Isacco Argiro (Mondrain 2007 [2008], 166)> (ff. 1r-151v, 153r-187v, 1. 13, 193r-204r, 1. 27, 206r-261v, 1. 18). 


\section{Decorazione}

Sono vergati in rosso i tituli, le iniziali minori, alcuni dei numerosi scolii a margine e le fasce ornamentali poste in apertura dei testi (cf. ff. 1r, 14r, 24v, 38v, 49r, 60v, 73r, $114 \mathrm{v}, 140 \mathrm{r}, 150 \mathrm{r}, 193 \mathrm{r}, 240 \mathrm{r}, 248 \mathrm{v}$ ). Argiro ha anche realizzato i diagrammi geometrici e le tavole astronomiche presenti nell'unità (cf. ff. 1v, 2rv, 3r, 4r, 8v, 9v, 17v, 18rv, 19r, 20-21r, 22r-23v, 24v, 25v, 28r, 30rv, 31v-34r, 35r-38r, 40r, 41v-42r, 42v-48r, 50r-51r, 52-57r, 58v-59r, 61r-64v, 65v-68r, 69v-71r, 72rv, 74r-75r, 79v-82r, 84r-85r, 90r-99v, 102v-103v, $106 \mathrm{r}-109 \mathrm{v}, 110 \mathrm{v}-111 \mathrm{r}, 112 \mathrm{r}-114 \mathrm{r}, 115 \mathrm{v}-121 \mathrm{r}, 122 \mathrm{r}-135 \mathrm{v}, 137 \mathrm{v}-139 \mathrm{v}, 142 \mathrm{r}, 143 \mathrm{r}-148 \mathrm{v}, 149 \mathrm{v}-151 \mathrm{v}$, 154r, 155r-161v, 163r-165v, 167rv, 168v-172v, 173v-175v, 176v-179v, 180v-185v, 193r-202r, 204r, 204r-208v, 209v-213v, 214v-216r, 217v-231r, 233r-236r, 237r-239v, 241r, 242r, 243r, 244r-247v, 248v-250v, 256r, 260v).

\section{Contenuto}

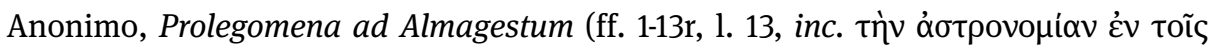

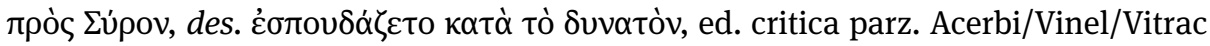
2010, 76-78, 120-132, 203-204; non critica parz. Tannery 1893-95 II, 3, 1. 18-15, 1. 17);

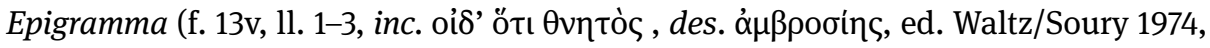
lib. IX, nr. 577); Pinax libri primi Syntaxeos Mathematicae (f. 13v, 1l. 4-12); Claudio

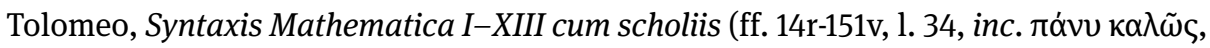

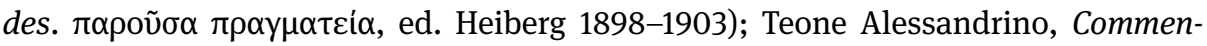

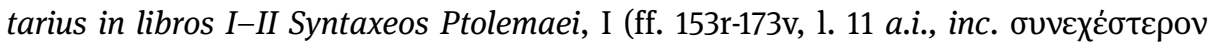

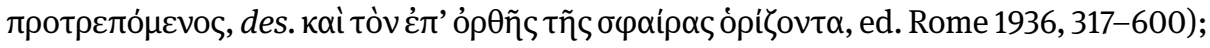

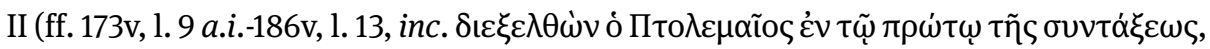

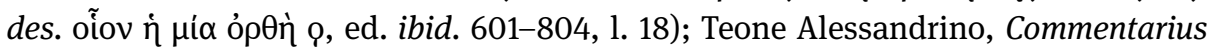

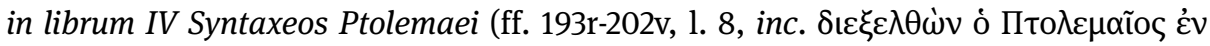

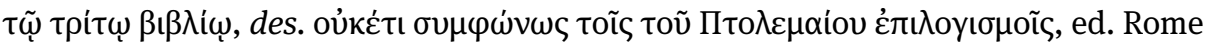
1943, 943-1085); Pappo Alessandrino, Commentarius in librum V Syntaxeos Ptolemaei

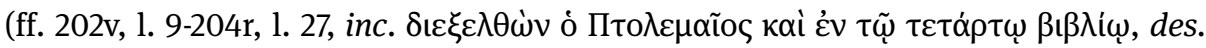

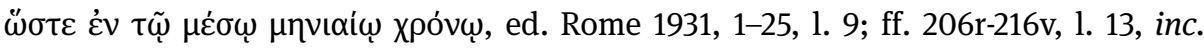

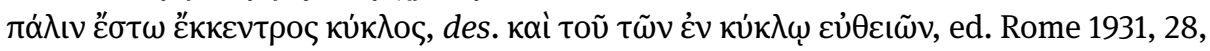
1. 13-169, 1. 10); Teone Alessandrino, Commentarius in libros VI-X et XII-XIII Synta-

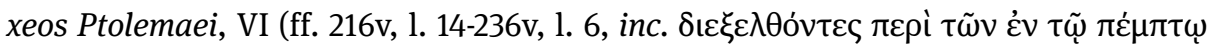

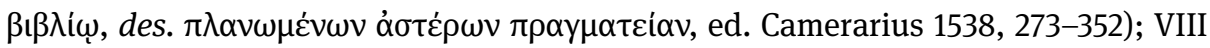

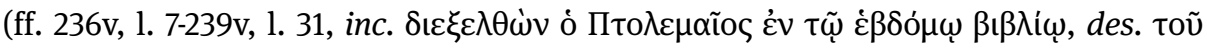

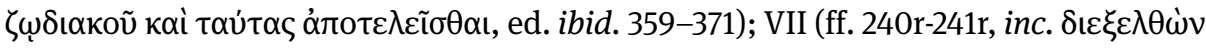

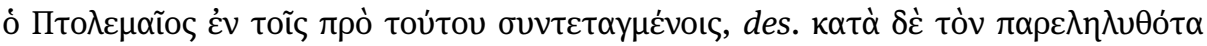

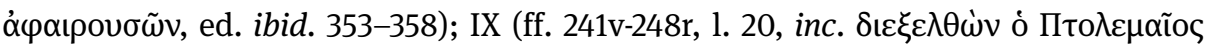

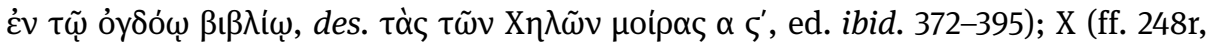

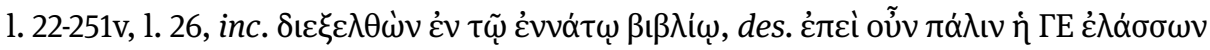




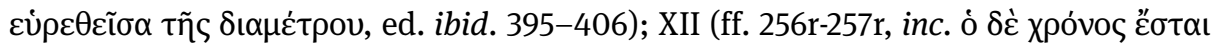

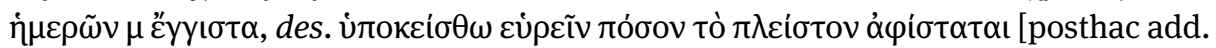

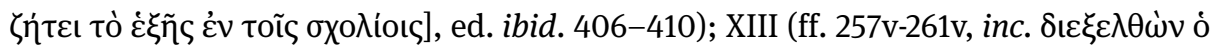

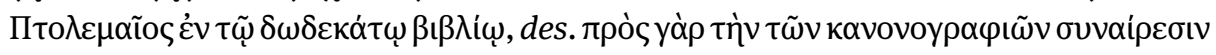

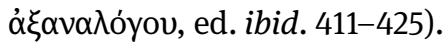

\section{II. ff. 266-288 (prima metà del XV sec.)}

Cart.; fil. 265, 266, 268, 270 simile a enclume (Br nr. 5956, 1450-1452), ff. 273, 276, 285, 286, 287 simile a fleur (Br nr. 6391, 1426-1434); 34-36 ${ }^{8}$ (288); num. nel marg. inf. del verso e del recto del

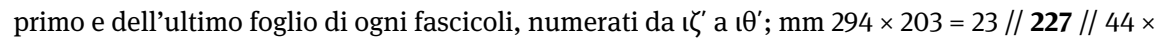
16 // 165 // 25; a piena pagina, 1l. 43, unità di rigatura mm 6; rigatura assente (f. 282).

\section{Scrittura}

La sezione è vergata da un'unica mano: <Bessarione (Mioni 1976, 282)> (ff. 266r-288r).

\section{Decorazione}

È vergato in rosso il titolo al f. 266r.

\section{Contenuto}

Nicola Cabasila, Commentarium in librum III Syntaxeos Ptolemaei (ff. 266r-285r, 1. 13,

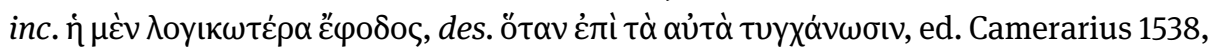

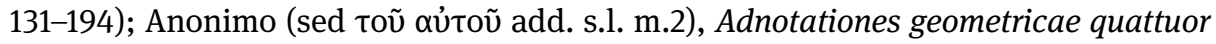

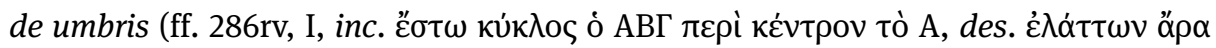

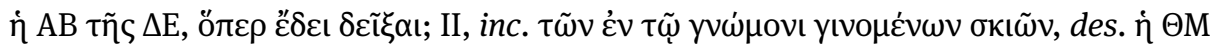

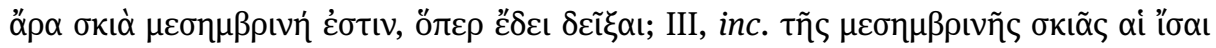

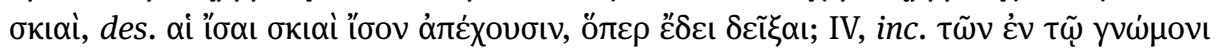

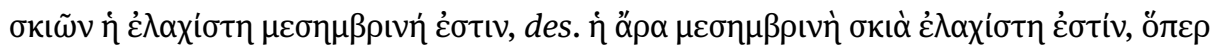

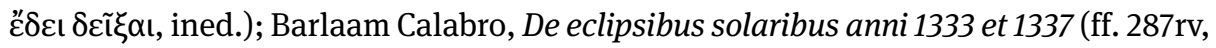

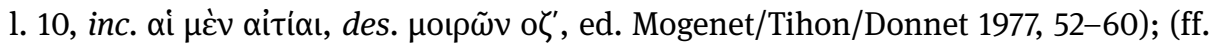

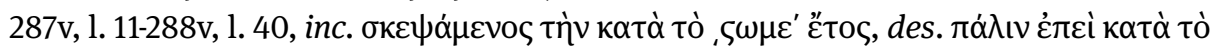

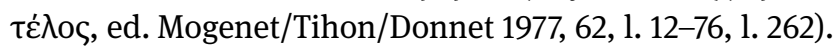




\section{Relazioni stemmatiche}

Apografo del Vat. gr. 1594 quanto al testo dell'Almagesto di Claudio Tolomeo (Heiberg 1907, XLI-XLII), trasmette insieme ad altri 5 testimoni la recensione bizantina dei Prolegomena ad Almagestum, redatta nei primi anni del XIV secolo (Acerbi/Vinel/Vitrac 2010, 68-69). Anche per i commentarii di Pappo e Teone all'Almagesto il codice (E) contiene una recensione bizantina del testo, come gli altri appartenenti al medesimo gruppo I (Rome 1943, 167). Il Marc. gr. Z. 310 fu utilizzato da Giorgio Trapezunzio per la sua traduzione latina dell'Almagesto (Acerbi apud Speranzi 2017, 184 n. 184)

\section{Storia del codice}

Il codice è stato identificato con l'esemplare nr. 247 dell'inventario redatto da Bessarione nel 1468 in occasione della donazione della propria collezione libraria alla basilica di San Marco a Venezia (Labowsky 1979, 167, Zorzi 2004, 20).

\section{Bibliografia}

\section{Cataloghi}

Mioni (1985) 20-22.

\section{Edizioni}

Acerbi/Vinel/Vitrac (2010); Camerarius (1538); Heiberg 1898-1903; Mogenet/Tihon/Donnet (1977); Rome (1931); Rome (1936); Rome (1943); Tannery (1893-1895) II; Walderus (1538); Waltz/Soury (1974).

\section{Studi}

Acerbi (2013) 141; Acerbi (2016) 160-161, 171-172, 175-176, 180, 187; Mioni (1976) 282; Mondrain

(2007) 166; Pérez Martín (2008) n. 127; Speranzi (2017) 184; Zorzi (2004) 20. 